\title{
Atenção à Saúde no Domicílio: modalidades que fundamentam sua prática
}

\section{Home Health Care: modalities that ground its practice}

Maria Ribeiro Lacerda

Doutora em Filosofia de Enfermagem. Professora Adjunta da Universidade Federal do Paraná (UFPR). Coordenadora do Núcleo de Estudos, Pesquisa e Extensão em Cuidado Humano de Enfermagem (Nepeche).

E-mail: lacerdaœmilenio.com.br

\section{Clélia Mozara Giacomozzi}

Enfermeira. Mestranda pelo Programa de Pós-graduação da UFPR. Membro do Nepeche. Bolsista Capes.

\section{Samantha Reikdal Oliniski}

Enfermeira. Mestre em Enfermagem pela UFPR.

Thiago Christel Truppel

Acadêmico do $10^{\circ}$ período do Curso de Graduação em Enfermagem da UFPR.

\section{Resumo}

A atenção domiciliar à saúde é um modelo em processo de expansão por todo o Brasil e desponta como um novo espaço de trabalho para os profissionais de saúde, tanto no âmbito público quanto no privado. Abrange quatro diferentes modalidades que foram selecionadas para estudo: atenção domiciliar, atendimento domiciliar, internação domiciliar e visita domiciliar. Cada uma dessas modalidades possui finalidades, objetivos e atividades específicas, o que torna necessária sua explicitação. Dessa forma, realizou-se esta revisão de literatura com o objetivo de identificar a bibliografia existente acerca dos termos utilizados para designar as modalidades. Considera-se necessário que os profissionais de saúde conheçam estas modalidades, pois provavelmente deparar-se-ão com elas, mesmo que não estejam atuando diretamente na atenção domiciliar à saúde. Reputa-se ainda que esse conhecimento seja importante para que a assistência prestada se dê de maneira mais adequada e correta, considerando as especificidades inerentes.

Palavras-chave: Assistência à saúde domiciliar; Serviços de assistência domiciliar; Prestação de cuidados de saúde. 


\section{Abstract}

Home health care is increasing all around Brazil and emerges as a new work place for health professionals, in public and in the private services. In Brazil home health care includes different kinds of assistance, four of those were studied in this project. Each one of the concepts or branches of home health care has specific purposes, objectives and activities. A review of the literature was undertaken in order to identify and to analyze these concepts. It is important that health professionals know these concepts because they will probably face them, even when they do not act directly in home care. This knowledge is also relevant for the quality and appropriateness of the provision of the services considering the inherent specificities.

Keywords: Home Care; Home Care Services; Delivery of Health Care.

\section{Introdução}

A atenção à saúde abrange dois modelos: o hospitalar e o domiciliar, sendo este último denominado de atenção domiciliar à saúde. 0 modelo domiciliar (re)surge em função das diversas alterações que a sociedade brasileira sofreu no decorrer dos anos como um modelo para o desenvolvimento de mudanças sociais e no sistema de saúde.

Dentre estas alterações, Duarte e Diogo (2000) apontam: a transição demográfica, que demonstra um envelhecimento populacional cada vez mais acentuado; a mudança no perfil epidemiológico da população, no qual se evidencia um aumento das doenças crônico-não transmissíveis; os custos do sistema hospitalar cada vez mais elevado; o desenvolvimento de equipamentos tecnológicos, que têm possibilitado maior taxa de sobrevida das pessoas; o aumento da procura por cuidados de saúde; o interesse dos profissionais de saúde por novas áreas de atuação; a exigência por maior privacidade, individualização e humanização da assistência à saúde, além da necessidade de maior integração da equipe profissional com o cliente e sua família.

Este modelo de atenção à saúde tem sido amplamente difundido no mundo e tem como pontos fundamentais o cliente, a família, o contexto domiciliar, o cuidador e a equipe multiprofissional.

Por se tratar de um modelo recente no Brasil, há muitos termos utilizados para sua designação, como: "home health care", "domiciliary care", atendimento domiciliar à saúde, atenção domiciliar, enfermagem domiciliar, visita domiciliária, "nursing home", "nursing care", "home care nursing", enfermagem residencial, cuidado domiciliar, internação domiciliar, cuidados médicos domiciliares, cuidados de saúde no domicílio, atendimentos médicos domiciliares e outros tantos. Muitos são utilizados como sinônimos, devido à tradução e à interpretação dos vocábulos "home care" ou "home health care" em português. Todavia, a oferta de serviços no domicílio, bem como a realização de atividades neste contexto, têm diferenças significativas.

Embora a atenção domiciliar à saúde esteja em processo de ascensão nas práticas de saúde, ela ainda não está completamente inserida nos sistemas de atendimento à saúde e na formação e/ou capacitação dos profissionais de saúde. Comporta diferentes mo- 
dalidades que são importantes para sua realização; para este texto serão consideradas as seguintes: a atenção, o atendimento, a internação e a visita domiciliares. Por terem perspectivas e serem atividades diferenciadas e concomitantemente complementares, estes conceitos devem ser diferenciados pelos profissionais que os executam, seja para que haja uma melhor articulação da teoria com a prática, para a adequação da prática profissional daqueles que já atuam na atenção à saúde domiciliar, seja para a formação dos novos profissionais de saúde.

Buscando a compreensão ampliada dos termos e de seus significados, bem como a sua utilização adequada pelos profissionais, realizou-se o presente estudo visando identificar a bibliografia existente acerca das concepções sobre termos, atenção, atendimento, internação e visita domiciliar.

\section{Metodologia}

Trata-se de uma revisão de literatura realizada por meio de pesquisa nas bases de dados Lilacs (Literatura Latino Americana e do Caribe em Ciências da Saúde) e BDENF (Base de Dados de Enfermagem). Para isso, foram selecionados os seguintes descritores de assunto: cuidados domiciliares de saúde, serviços de assistência domiciliar e reforma dos serviços de saúde. Também foram utilizadas as palavras: cuidado domiciliar, visita domiciliária, atenção à saúde, atendimento domiciliar e assistência domiciliar.

Utilizou-se a biblioteca virtual Bireme, na qual a pesquisa foi delimitada para artigos publicados a partir do ano de 1999. O período da realização da busca foi contínuo, e o intervalo considerado para a realização deste trabalho compreendeu de janeiro de 2005 a maio de 2006. Foram diversos os achados nesta busca, sendo mais utilizados os artigos clássicos como tipo de publicações. Por meio dos artigos encontrados, pôde-se, ainda, buscar outras referências e documentos que neles constavam.

Outras formas de obtenção de dados também foram utilizadas, como: a biblioteca do setor de Ciências da Saúde da Universidade Federal do Paraná (UFPR) e as publicações a que tivemos acesso por meio da utilização da internet, em sites oficiais e de outros estudiosos da área.

Assim, foram utilizadas fontes diversas de infor- mação, como artigos publicados em periódicos, arquivos eletrônicos e livros, com o objetivo de embasar o conhecimento acerca dos diferentes conceitos utilizados na atenção domiciliar à saúde.

\section{Revisão de Literatura}

Considerando que as modalidades relativas à atenção domiciliar à saúde possuem diferentes percepções e abordagens, buscou-se utilizar distintos autores para conhecer quais são as concepções existentes no meio científico e profissional sobre o tema.

Conforme comentado anteriormente, é necessário que os profissionais de saúde tenham clareza em relação aos termos da atenção domiciliar à saúde, para que possam fundamentar suas práticas e para que tenham uma visão unificada, e, assim, obtenha-se maior êxito na realização de seus objetivos.

A seguir, serão apresentadas definições das quatro modalidades da atenção domiciliar à saúde: atenção domiciliar, atendimento domiciliar, internação domiciliar e visita domiciliar. Essa divisão é proposta pelos autores com base nos termos observados em publicações, sendo concordante com a Resolução RDC $\mathrm{n}^{0}$. 11, de 26 de janeiro de 2006 da Anvisa e como com o Ministério da Saúde, em Documento Preliminar publicado em 2004. Esses dois documentos oficiais dividem a atenção domiciliar à saúde em: atenção domiciliar, assistência domiciliar e internação domiciliar, aos quais acrescentamos a modalidades da visita domiciliar (Brasil, 2006; Brasil, 2004).

\section{Atenção Domiciliar}

A atenção domiciliar é a modalidade de maior amplitude dentre as quatro citadas. Ela é definida pela Brasil (2006) como um termo genérico, que envolve ações de promoção à saúde, prevenção e tratamento de doenças e reabilitação, desenvolvidas em domicílio.

As ações de saúde são realizadas no domicílio do paciente por uma equipe multiprofissional, a partir do diagnóstico da realidade em que o paciente está inserido, visando a promoção, manutenção e/ou restauração da saúde (Duarte; Diogo, 20oo), portanto, é uma atividade que envolve não só os diferentes profissionais da área da saúde, como também o cliente e sua família, visando ao estabelecimento da saúde 
como um todo. Permite que os profissionais desenvolvam atividades de modo que o cliente perceba que a sua participação no processo saúde-doença é de fundamental importância, pois é ele (o cliente) que poderá diminuir ou até mesmo eliminar os fatores que colocam em risco sua saúde, não bastando apenas à informação veiculada pelos profissionais.

Mazza (2004) considera a atenção domiciliar à saúde como "um dos meios de se obter do indivíduo, da família e da comunidade sua participação no planejamento, [na] organização, [na] operação e [no] controle" dos cuidados primários em saúde, fazendo uso dos recursos locais disponíveis.

Complementa-se que a atenção domiciliar envolve a prática de políticas econômicas, sociais e de saúde, para reduzir os riscos de os indivíduos adoecerem; a fiscalização e o planejamento dos programas de saúde; e a execução das atividades assistenciais, preventivas e educativas. Assim, abrange desde a promoção até a recuperação dos indivíduos acometidos por um agravo e que estão sediados em seus respectivos lares.

Essa modalidade abrange todas as outras, isto é, o atendimento, a internação e a visita domiciliária, que possibilitam a realização e a implementação da atenção domiciliar, de modo que todas as ações que possam vir a influenciar o processo saúde-doença das pessoas.

Para o modelo americano, a atenção domiciliar à saúde pode ser compreendida como "home health care”, sendo o cuidado à saúde proporcionado às pessoas no próprio domicílio de forma apropriada e de alta qualidade, com relação ao custo-benefício compatível com a vida dos indivíduos, que devem manter sua autonomia, independência e melhor qualidade de vida (Word Health Organization, 1999).

\section{Atendimento Domiciliar}

Este termo é utilizado por alguns autores para designar atividades nomeadas como assistência domiciliar ou, por outros autores, como cuidado domiciliar. Assim, considera-se esses três termos sinônimos e representantes de uma mesma modalidade da atenção domiciliar à saúde. Segundo a Anvisa (Brasil, 20o6), assistência domiciliar (ou atendimento) é um conjunto de atividades de caráter ambulatorial, programadas e continuadas, desenvolvidas em domicílio.
Podemos compreender essa expressão como "home care" em outros países. No sistema de saúde canadense é a provisão de uma disposição de serviços de saúde e sociais designados a dar suporte aos pacientes em suas próprias casas (Wealth Canada, 1997). O objetivo é permitir que indivíduos que necessitem de cuidados para circunstâncias agudas ou crônicas de saúde recebam o tratamento da alta qualidade no domicílio ou na comunidade, ou facilidades a longo prazo do cuidado, sendo que a sustentação ou cuidado para os amigos e a família, que fornecem o cuidado para seus familiares doentes é também importante. $\mathrm{Na}$ Coréia o "home care" esta baseado em uma perspectiva do setor público de saúde e as ações de saúde no domicílio junto à família e à comunidade se consolidam para pacientes com doenças crônicas e de longo tratamento, que estejam sem hospitalização (Ryu, an, Koabyashi, 2004).

Melies (1997) sugere que o cuidado domiciliar "home care" - seja visto como um conceito relacionado ao fenômeno de "home care", que pode ser compreendido como as atividades que representam o todo das atividades de cuidado, desde as de prevenção primária até os cuidados paliativos para o fim da vida. Assim, representam um extenso campo de atividades de cuidado.

O cuidado dos profissionais de saúde para a pessoa em sua própria casa, com o objetivo final de bemestar, além de contribuir para a qualidade de vida e para o funcionamento do estado de saúde, substitui o cuidado hospitalar, esta é uma compreensão do sistema de saúde sueco. (Thomé, Dykes e Hallberg, 2003).

$\mathrm{O}$ atendimento domiciliar representa uma estratégia de atenção à saúde, que engloba mais do que o fornecimento de um tratamento padrão. É um método aplicado ao cliente com o objetivo de enfatizar sua autonomia e realçar suas habilidades em seu próprio ambiente - o domicílio (Duarte e Diogo, 20oo). Ele envolve ações menos complexas, multiprofissionais ou não, que podem ser comparadas a um "consultório em casa" (Tavolari e col., 200o). Segundo Paskulin e Dias (2002), o atendimento domiciliar pode propiciar um contato mais estreito dos profissionais de saúde com o paciente e seus familiares em seu próprio meio, podendo este momento ser útil para uma avaliação das condições que o cercam, por vezes, de grande importância para o sucesso do acompanhamento. 
Compreende-se que o atendimento domiciliar envolve a realização de ações educativas, orientação, demonstração de procedimentos técnicos a serem delegados ao cliente ou ao seu cuidador, bem como a execução destes procedimentos pela equipe multiprofissional no domicílio do cliente.

$\mathrm{O}$ atendimento domiciliar é, portanto, um conjunto de ações que busca a prevenção de um agravo à saúde, a sua manutenção por meio de elementos que fortaleçam os fatores benéficos ao indivíduo e, concomitantemente, a recuperação do cliente já acometido por uma doença ou seqüela.

Evidencia-se com isso que o atendimento domiciliar compreende todas as ações, sejam elas educativas ou assistenciais, desenvolvidas pelos profissionais de saúde no domicílio do cliente, direcionadas a ele próprio e/ou a seus familiares. Desse modo, abrange tanto atividades simples como as mais complexas, incluindo, assim, as modalidades visita e internação domiciliar.

\section{Internação Domiciliar}

A internação domiciliar é uma atividade continuada, com oferta de tecnologia e de recursos humanos, equipamentos, materiais e medicamentos, para pacientes mais em estados mais complexos, que demandam assistência semelhante à oferecida em ambiente hospitalar (Ribeiro, 2004).

Para Lacerda (200o), a internação domicilia é a prestação de cuidados sistematizados de forma integral e contínua no domicílio, com supervisão e ação da equipe de saúde específica, personalizada, centrada na realidade do cliente, envolvendo a família e, podendo ou não, utilizar equipamentos e materiais. A autora pontua ainda que, nessa modalidade, o cliente recebe cuidados e orientações sobre várias ações, pois está com o estado de saúde alterado, precisando de acompanhamento profissional.

O uso da palavra internação requer cautela, pois pode incorrer em apropriação da terminologia e da compreensão utilizada em instituições hospitalares, não considerando a especificidade que o domicílio apresenta (Lacerda, 200o).

A internação domiciliar, segundo Tavolari, Fernandes e Medina (200o), refere-se ao cuidado intensi- vo, contínuo e multiprofissional, desenvolvido em casa. Requer aparato tecnológico semelhante ao existente na estrutura hospitalar, pois o cuidado é direcionado a doentes com complexidade moderada ou alta, no entanto, esses recursos tecnológicos são solicitados de acordo com a necessidade do cliente.

Dal Ben (2001) afirma que nesta modalidade o acompanhamento contínuo, ou seja, diário e até, às vezes, ininterrupto, pode ser de 24, 12, 8 ou 6 horas de assistência de enfermagem. A autora refere ainda que "é fundamental o suporte contínuo com uma central de atendimento para solução de emergência, durante 24 horas, com médico e enfermeiro disponíveis para orientar e atender às necessidades do paciente".

A internação domiciliar, portanto, é uma modalidade que tem se revelado uma opção segura e eficaz, direcionada a pacientes portadores de doenças crônicas ou agudas (Freitas e col., 200o). Está adquirindo cada vez mais importância nas distintas organizações sanitárias e sociais, acompanhada por estudos que prevêem um aumento dos serviços de atendimento domiciliar, assinalando que diferentes setores colocam a internação domiciliar como alternativa viável e promissora (Lacerda, 1999).

Na França, a internação domiciliar é uma alternativa assistencial do setor de saúde que consiste em um modelo organizado capaz de dispensar um conjunto de cuidados e atenção de médicos, enfermeiras, tanto em qualidade como em quantidade, para pacientes em seu domicílio, que não precisam da infra-estrutura hospitalar, mas necessitam de vigilância ativa e assistência completa (Nogueira, e col., 200o).

Para Cotta (2001), é importante salientar que a internação domiciliar deve ter caráter transitório e que a equipe de saúde que atende o paciente no domicílio esteja dotada de meios tecnológicos necessários e que visitem regularmente a residência do paciente para fazer o diagnóstico e prover o tratamento e os cuidados.

Em um documento preliminar do Ministério de Saúde brasileiro sobre as diretrizes para a atenção domiciliar no sistema único de saúde (Brasil, 2004), há as seguintes ações que podem ser realizadas pela internação domiciliar: procedimentos terapêuticos, educação sanitária, cuidados paliativos e visitas de moni- 
toramento, pautando-se pelo cuidado integral, por ações inter- e transdisciplinares, devendo ser consideradas as condições locais, as questões habitacionais, sociais, culturais, a formação de equipes, a rede básica e a decisão do gestor local de saúde.

\section{Visita Domiciliar}

O conceito de visita domiciliar parece ser o mais difundido no sistema de saúde brasileiro e nas praticas de saúde na comunidade. Ribeiro (2004) o considera um contato pontual de profissionais de saúde com as populações de risco, enfermos e seus familiares para a coleta de informações e/ou orientações. Na visita domiciliar são desenvolvidas ações de orientação, educação, levantamento de possíveis soluções de saúde, fornecimento de subsídios educativos, para que os indivíduos atendidos tenham condições de se tornar independentes (Mazza, 2004).

As orientações realizadas dizem respeito a saneamento básico, cuidados com a saúde, uso de medicamentos, amamentação, controle de peso, ou qualquer coisa que diga respeito àquele indivíduo, à família e à comunidade em que vivem (Jacob, 2001).

Segundo Lacerda (1999), a visita é realizada pelos profissionais de saúde e/ou equipe na residência do cliente, com o objetivo de avaliar as demandas exigidas por ele e seus familiares, bem como o ambiente onde vivem, visando estabelecer um plano assistencial, geralmente programado com objetivo definido.

Ela pode ser utilizada, ainda, como atividade de subsidio de intervenção no processo saúde-doença de indivíduos ou o planejamento de ações visando à promoção da saúde coletiva. Deve compreender ações sistematizadas, que se iniciam antes da visita e continuam após ela. (Freitas e col., 200o).

Conclui-se, portanto, que a visita domiciliar é uma forma de assistência domiciliar à saúde, que dá subsídios para a execução dos demais conceitos desse modelo assistencial. É, por intermédio da visita, que os profissionais captam a realidade dos indivíduos assistidos, reconhecendo seus problemas e suas necessidades de saúde.

No Japão, as atividades de visita domiciliar são realizadas pelas enfermeiras que a definem como ati- vidade que orienta o paciente e a família nas atividades da vida diária e na provisão de um cuidado individual ao paciente, sendo estes cuidados ofertados a pacientes idosos, a pacientes que necessitam de cuidados especiais e de tecnologia avançada, a pacientes terminais e a pacientes com doenças mentais (Murashima e col., 2002).

\section{Uma Visão Global dos Conceitos}

A internação domiciliar é a mais específica modalidade da atenção domiciliar à saúde, envolvendo a presença contínua de profissionais no domicílio e o uso de equipamentos e materiais. Ela é uma forma de operacionalizar o atendimento domiciliar, assim como utiliza a visita domiciliar como estratégia de realização desta atividade.

A visita domiciliar possui um caráter mais pontual, de contato com os profissionais, que observam a realidade do paciente em seu domicílio, isto é, seu contexto domiciliar, seja de estrutura física e material ou de relações pessoais intra-familiares; em que também podem realizar orientações.

0 atendimento domiciliar é uma modalidade um pouco mais ampla, que envolve atividades profissionais realizadas diretamente no domicílio das pessoas, envolvendo o cliente e sua família. Conforme dito anteriormente, pode ser operacionalizado pela internação ou pela visita domiciliar; mas, ainda, pode ser simplesmente atendimento domiciliar. Isso porque os profissionais podem realizar atividades e procedimentos no domicílio, sem configurar o tratamento intensivo da internação domiciliar ou os objetivos de educação e levantamento de dados da visita domiciliar, constituindo atendimento domiciliar propriamente dito.

A atenção domiciliar à saúde, além de envolver as atividades profissionais da internação, da visita ou do atendimento domiciliares, engloba práticas de políticas de saúde, saneamento, habitação e educação, dentre outras; dependendo de diferentes esferas de atuação para a saúde dos grupos sociais.

Assim, as modalidades apresentadas possuem diferenças entre si, mas são complementares e interdependentes, como pode ser observado na representação esquemática da figura 1. 
Figura I - Representação esquemática da atenção à saúde domiciliar e suas respectivas modalidades. Elaborado pelos autores.

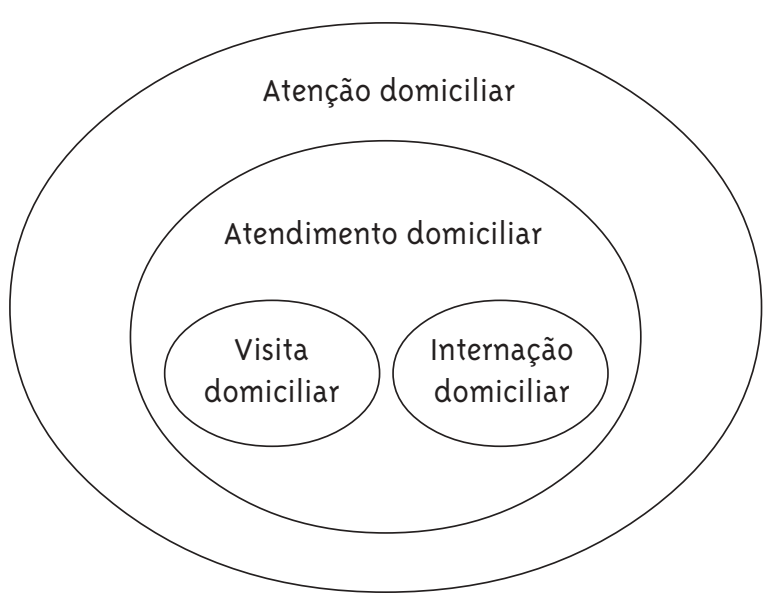

\section{Considerações Finais}

Há uma complementaridade entre as modalidades da atenção domiciliar, atendimento domiciliar, visita e internação domiciliar. Cada uma possui peculiaridades e características que as distinguem, e devem ser estabelecidas de maneira clara pelos profissionais de saúde, não apenas por questões de operacionalização dos serviços de saúde, mas para sua orientação na execução das práticas profissionais.

Para que o modelo de atenção à saúde domiciliar ocorra de forma a efetivar seus preceitos, deve-se lançar mão de suas modalidades para que a prática assistencial seja planejada, sistematizada, organizada e documentada. Esse último aspecto merece ênfase, pois é muito importante para a avaliação desse modelo assistencial e seu constante aperfeiçoamento.

Este artigo pretende propor uma reflexão sobre as modalidades da atenção domiciliar à saúde, de modo a auxiliar os profissionais na aquisição de subsídios teóricos para uma prática fundamentada. Deste modo, afirma-se que a atenção domiciliar é a modalidade mais ampla, envolvendo a vigilância à saúde dos indivíduos, de modo a promover, manter e/ou restaurar a saúde da população. 0 atendimento é a execução de qualquer atividade profissional direta à saúde dos indivíduos, ao passo que a internação domiciliar é uma prática mais intensiva e complexa no domicílio do cli- ente e a visita domiciliar é uma forma de avaliação dos indivíduos, da família e do domicílio, sendo, os dois últimos, uma forma de atendimento domiciliar.

Não se pretende exaurir a discussão sobre o tema. Tem-se o intuito, sim, de chamar a atenção dos profissionais da área da saúde para uma nova concepção, para uma estratégia de assistência à saúde inovadora, pautada em uma percepção diferenciada sobre o processo saúde-doença, que contempla o indivíduo e seus familiares em seu domicilio, em cujo espaço dimensões sociais e afetivas se efetuam.

Com o ressurgimento dessa modalidade, há a necessidade de os profissionais buscarem atualização e qualificação profissional, para atuar nesse campo de trabalho, espaço que trará grandes perspectivas para o profissional, visto os fatores que favorecem a implementação desse modelo.

Por fim, existe a certeza de que os maiores beneficiados da atenção domiciliar à saúde serão os clientes e seus familiares, porque o cuidado passará a ser individualizado, humanizado, distante dos riscos iatrogênicos, pertinentes ao contexto hospitalar e, acima de tudo, contará com a participação do principal cuidador: o familiar ou o significante.

\section{Referências}

BRASIL. Ministério da Saúde. Agência Nacional de Vigilância Sanitária. Resolução RDC n ${ }^{0}$ 11, de 26 de janeiro de 2006. Diário Oficial da União, Poder Executivo, Brasília, DF, 30 jan. 2006 Disponível em: <http:// e-legis.anvisa.gov.br/leisref/public/showAct.php?id= $20642 \&$ word=rdc\%202006\%2odomiciliar $>$. Acesso em: 20 mar. 2006

BRASIL. Ministério da Saúde. Diretrizes para a atenção domiciliar no sistema único de saúde. Brasília, DF, 2004

COTTA, R. M. M. et al. La hospitalización domiciliaria: antecedentes, situación actual y perspectivas. Revista Panamericana de Salud Pública, Washington, DC, v. 10, n. 1, p. 45-55, jul. 2001.

DAL BEN, L. W. Home care. Médicis, São Paulo, n. 11, p. 14-17, jul./ago. 2001.

DUARTE, Y. A. O.; DIOGO, M. J. E. Atendimento domiciliar: um enfoque gerontológico. São Paulo: Atheneu, 2000. 
FREITAS, A. V. S.; BITTENCOURT, C. M. M.; TAVARES, J. L. Atuação da enfermagem no serviço de internação domiciliar: relato de experiência. Revista Baiana de Enfermagem, Salvador, v.13, n. 1/2, p.103-107, abr./out. 2000.

JACOB, W. Digerir as diferenças. Médicis, São Paulo, n. 13, p. 3-8, nov./dez. 2001.

LACERDA, M. R. Internação domiciliar. In: CONGRESSO BRASILEIRO DE ENFERMAGEM, 51., 1999, Florianópolis. Anais... Florianópolis: ABEn, 1999.

LACERDA, M. R. Tornando-se profissional no contexto domiciliar: vivência do cuidado da enfermeira. 2000. Tese (Doutorado em Filosofia da Enfermagem) - Universidade Federal de Santa Catarina, Florianópolis, 2000.

MAZZA, M. M. P. R. A visita domiciliária como instrumento de assistência de saúde. Disponível em: <http:/ /www.fsp.usp.br/MAZZA.htm>. Acesso em: 1 jun. 2004.

MURASHIMA, S. et al. Home care nursing in Japan: a challenge for providing good care at home. Public Health Nursing. v. 19, n. 2, p. 94-103, 2002.

NOGUEIRA, J. M. S. et al. Enfermos crônicos domiciliares: valorização integral físico-cognitiva e caídas durante 3 anos de seguimento. Atencion Primária, v. 25, n. 3, p. 16-17, 2000.

PASKULIN, L. M.; DIAS, V. R. Como é ser cuidado em casa: as percepções os clientes. Revista Brasileira de Enfermagem, Brasília, DF, v. 55, n. 2, p. 140-145, mar./ abr. 2002.
RIBEIRO, C. A. Assistência domiciliar: uma 'nova' modalidade de atenção à saúde. Disponível em: <http:/ /www.unimeds.com.br/conteúdo/MEo1.htm>. Acesso em: 5 ago. 2004.

RYU, H.; AN, J.; KOABYASHI, M. Home health nursing care agenda based on health policy in Korea. Nursing and Health Sciences, v. 7, n. 2, p. 92-98, 2005.

TAVOLARI, C. E. L.; FERNANDES, F.; MEDINA, P. $O$ desenvolvimento do 'Home Health Care' no Brasil. Revista de Administração em Saúde, São Paulo, v. 3, n. 9, p. 15-18, out./dez. 2000.

THOMÉ, B.; DYKES, A. K.; HALLBERG, I. R. Home care with regard to definition, care recipients, content and outcome: systematic literature review. Journal of Clinical Nursing, Oxford, v. 12, n. 6, p. 860-872, 2003.

WEALTH CANADA. Policy and Consultation Branch. National health expenditures in Canada 1975-1996: fact sheets. Ottawa, 1997. Disponível em: <http:// socialpolicy.ca/cush/m7/healthfact.pdf $>$. Acesso em: 7 jul. 2006.

WORD HEALTH ORGANIZATION. Home-based and long-term care: home care issues at the approach of the 21th century from a World Health Organization perspective - a literature review. Genebra, 1999. (WHO/ HSC/LTH/99/1). 